\title{
Favorable Response
}

National Cancer Institute

\section{Source}

National Cancer Institute. Favorable Response. NCI Thesaurus. Code C123584.

No new lesions(s) and a decrease in tumor size, or a reduction in central necrosis or attenuation in one or more enhancing lesions, based on pre-defined thresholds. 